\title{
Hundred Years of Einstein's Cosmological Constant
}

\author{
Kenath Arun $^{1,2^{*}}$, S B Gudennavar ${ }^{1}$, Kiren O V ${ }^{3}$ and C Sivaram ${ }^{4}$ \\ ${ }^{1}$ Department of Physics and Electronics, Christ University, Bengaluru, India \\ ${ }^{2}$ Department of Physics, Christ Junior College, Bengaluru, India \\ ${ }^{3}$ Department of Physics, St. Josephs Indian Composite PU College, Bengaluru, India \\ ${ }^{4}$ Indian Institute of Astrophysics, Bengaluru, India \\ *Email: kenath.arun@cjc.christcollege.edu
}

\begin{abstract}
It was little over a hundred years ago that Einstein introduced the 'cosmological constant' in his General Theory of Relativity in order to obtain a static universe, to conform to the philosophical view of the universe at that time. What Einstein subsequently dubbed as the 'biggest blunder' of his life (after Hubble's discovery of the expanding universe) has come back in vogue in cosmology. Here we look at the evolution of the concept of the cosmological constant from its inception to it possibly making up close to about $70 \%$ of the energy density of the universe.
\end{abstract}

Keywords: General Theory of Relativity, cosmological constant, expanding universe, cosmology, dark energy.

\section{Introduction}

On February 4 1917, Einstein wrote to Ehrenfest (quoted in The Invented Universe [1]), "I have ... again perpetrated something about gravitation theory which somewhat exposes me to the danger of being confined in a madhouse." He revealed these maddening thoughts four days later to the Royal Prussian Academy of Science, and these were published a week later by the Academy [2]. Thus began modern relativistic cosmology.

\section{$2 \quad$ Why and What Did Einstein Introduce?}

While formulating the principle of equivalence, i.e. an accelerated frame is locally equivalent to a uniform gravitational field (this explains why all bodies sufficiently close to each other, fall with the same acceleration in the earth's field), Einstein realized that gravity can be described entirely by the geometry of space-time [3-5]. Unlike the flat Minkowskian space-time of Special Relativity (SR) (frames moving with uniform relative velocity in which laws of physics are invariant in all inertial frames), accelerated frames (or equivalently gravity) is described by curved space-time General Relativity (GR). The curvature is caused by the distribution of matter, or more precisely energy includes mass energy [6].

'Space-time tells matter how to move; matter tells space-time how to curve' [7], i.e. particles follow the geodesics or shortest paths of the curved space-time caused by a distribution of matter (like a point mass or a field). So in General Relativity, gravity is not a force due to matter like in Newtonian theory, but 'gravitational' motion is a consequence of particles following a curved path in space-time with curvature, due to equivalent energy. In the absence of matter there is no gravity and we have flat spacetime. The field equation for gravity in the Newtonian theory is given by Poisson's equation [5]

$$
\Delta^{2} \phi=4 \pi \mathrm{G}
$$

where $G$ is Newton's gravitational constant, $\phi$ is the scalar gravitational potential and $\rho$ the matter density which is the source for the scalar gravitational potential.

In Einstein's GR, the potentials are given by the metric tensor, which is a purely geometric quantity, describing how distances between points in the curved space are evaluated. For instance, in Euclidean space in Cartesian coordinates, we represent the infinitesim $\phi$ al distance, $d s$, between two points as 
$d s^{2}=1 d x^{2}+1 d y^{2}+1 d z^{2}$. The coefficients in front of $d x^{2}, d y^{2}, d z^{2}$ are called components of the metric tensor, i.e. $\mathrm{g}_{11}=1, \mathrm{~g}_{22}=1, \mathrm{~g}_{33}=1, \mathrm{~g}_{i j}=0$ for $i \neq j$. In spherical coordinates we have, $d s^{2}=1 d r^{2}+r^{2} d \theta^{2}+r^{2} \sin ^{2} \theta d \phi^{2} \quad$ and $\mathrm{g}_{11}=1, \mathrm{~g}_{22}=r^{2}, \mathrm{~g}_{33}=r^{2} \sin ^{2} \theta$.

Similarly the infinitesimal 'distance' in flat space-time is $d s^{2}=c^{2} d t^{2}-1 d x^{2}-1 d y^{2}-1 d z^{2}$ with a corresponding diagonal metric tensor $\mathrm{g}_{\alpha \beta}=\left(c^{2},-1,-1,-1\right)$. In general the metric tensor (a second order tensor) is notated as

$$
d s^{2}=\sum \sum \mathrm{g}_{\alpha \beta} d x^{\alpha} d x^{\beta}=\mathrm{g}_{\alpha \beta} d x^{\alpha} d x^{\beta}
$$

(repetition of $\alpha, \beta$ denoting summation)

The motivation behind Einstein's GR is that a matter distribution distorts space-time around it. Thus the flat space-time metric in spherical coordinates

$$
d s^{2}=c^{2} d t^{2}-d r^{2}-r^{2} d \theta^{2}-r^{2} \sin ^{2} \theta d \phi^{2}
$$

is modified by the presence of a point mass $M$, to the metric (this is the well-known Schwarzschild metric)

$$
d s^{2}=\left(1-\frac{2 G M}{c^{2}}\right) c^{2} d t^{2}-\left(1-\frac{2 G M}{c^{2}}\right)^{-1} d r^{2}-r^{2} d \theta^{2}-r^{2} \sin ^{2} \theta d \phi^{2}
$$

There are other such metrics for different matter sources, which also involve a modification of the flat space-time metric. The Poisson's equation in GR maybe expected to be given by

$$
\nabla^{2} \mathrm{~g}_{\alpha \beta}=4 \pi G \rho
$$

which is however incorrect, since the $\mathrm{g}_{\alpha \beta}$ in 4 dimensional space-time has actually ten components, and we would also expect the source term on the RHS to be a second order tensor, instead of just the scalar $\rho$ (matter density).

Indeed we do have a second order tensor, namely the energy momentum tensor $T_{\alpha \beta}$, which includes pressure terms, shear stress, etc. So the relativistic analogous of Poisson's equation in Newtonian gravity, hereafter referred to as Einstein's filed equations, would be expected to resemble

$$
\nabla^{2} \mathrm{~g}_{\alpha \beta}=4 \pi G T_{\alpha \beta}
$$

Thus in General Relativity, all sources of energy (pressure, stress, viscosity, etc.) contribute to gravity, not just the mass density $\rho$. For a perfect fluid, $T_{\alpha \beta}$ includes just $\rho$ and three pressure components, $P_{i}$.

Since in GR gravitational effects are related to the curvature of space-time we now discuss the geometric analogue of the second derivative of the metric tensor. On a plane (zero curvature or infinite radius of curvature), a triangle has the sum of its angles equal to $\pi$ radians (or $180^{\circ}$ ) (Euclidean geometry). However, a triangle drawn on a sphere (spherical triangle) has the sum of all its angles $>\Pi$. The difference over $\Pi$ is proportional to area of the triangle divided by radius of sphere squared. If a vector is transported over a triangular area covering an octet of the sphere, so that at all points it is kept parallel to itself (parallel transport), then on returning to the original point after moving along the area, it will change direction by an angle of $\Pi / 2$.

The curvature is then defined as the ratio of direction (angle) change to the area traversed by the vector, i.e.

$$
R=\frac{\pi / 2}{1 / 8\left(4 \pi a^{2}\right)}=\frac{1}{a^{2}}
$$

The sphere is uniformly curved. For a plane, the radius $a \rightarrow \infty \Rightarrow R=0$. In a plane if we parallel transport a vector, its direction remains unchanged, whereas in a curved surface it does not. For a nonspherical area, $d x^{\alpha} d x^{\beta}$, the change in direction of the vector is given by

$$
\Delta V^{\mu}=R_{\alpha \beta \gamma}^{\mu} d x^{\alpha} d x^{\beta} n^{\gamma}
$$


where $n^{\gamma}$ is the unit normal vector, $R_{\alpha \beta \gamma}^{\mu}$ is called the Riemann tensor, a fourth order curvature tensor and is composed of the second derivative of the metric tensor. As the metric tensor (second order tensor) is the analogue of potential in Newtonian gravity, to replace $\nabla^{2} \phi$, we need a tensor containing second derivative of metric tensor $\mathrm{g}_{\alpha \beta}$. Such a tensor is $R_{\alpha \beta \gamma}^{\mu}$, but the source term is $T_{\alpha \beta}$, a second order tensor, so left hand side also must have second order. This is the Ricci tensor obtained by contracting the Riemann curvature tensor using the metric tensor as: $\mathrm{g}^{\mu v} R_{\alpha \beta \mu v}=R_{\alpha \beta}$. So we may expect the field equations to be

$$
R_{\alpha \beta}=\kappa T_{\alpha \beta}
$$

where $\kappa$ is a constant.

For any constant physical quantity, its derivatives must vanish. Conservation of the energy momentum tensor in GR is written as $D_{\beta} T^{\alpha \beta}=T_{; \beta}^{\alpha \beta}=0 \quad\left(D_{\beta}\right.$ is called the covariant derivative, as it transforms as a tensor, i.e. unlike the usual partial derivative). $T_{; \beta}^{\alpha \beta}=0$ and equation (9) implies that $R_{; \beta}^{\alpha \beta}=0$, but it turns out $R_{; \beta}^{\alpha \beta} \neq 0$. Earlier papers of Einstein and Grossman had the erroneous field equations in equation (9). However, Einstein then found that $\left(R_{\alpha \beta}-\frac{1}{2} \mathrm{~g}_{\alpha \beta} R\right)_{; \beta}=G_{\alpha \beta ; \beta}=0$. Here $R$ is the curvature scalar obtained by contracting the Ricci tensor, $R=g^{\alpha \beta} R_{\alpha \beta}$. In the case of a sphere, $R$ is a constant and can be related to a constant density as

$$
R=\frac{8 \pi G \rho}{c^{2}}
$$

In the case of zero density $(\rho=0) R=0$, i.e. there is no curvature, i.e. flat space-time. $G_{\alpha \beta}$ is the Einstein tensor and is the appropriate tensor to use in the field equations. Thus, $G_{\alpha \beta}=\kappa T_{\alpha \beta}$ are the GR field equations. Einstein also showed that if one adds a $\Lambda g_{\alpha \beta}$ term to $G_{\alpha \beta}$, (where $\Lambda$ is a constant having dimensions of curvature), one still gets

$$
\left(G_{\alpha \beta}+\Lambda g_{\alpha \beta}\right)_{; \beta}=0
$$

So the more general field equations are

$$
G_{\alpha \beta}+\Lambda g_{\alpha \beta}=\kappa T_{\alpha \beta}
$$

This form of the field equations was justified by Hilbert's variational principle, with the action: $\int(R+2 \Lambda) \sqrt{-g} d^{4} x$, which indeed gives the field equation (equation (12)). Field equations in physics for scalar or vector field can be obtained from a variational principle where an action is written involving the field and its derivative.

In classical mechanics for example it is well known that the Lagrangian $L=f(x, \dot{x}, t)$ being a function of position $(x)$, velocity $(\dot{x})$ and time $(t)$ gives rise to equations of motion $\frac{\partial L}{\partial x}-\frac{d}{d t}\left(\frac{\partial L}{\partial \dot{x}}\right)=0$. L can be functions of fields and their derivatives instead of position and velocity.

Spurred by the successes of his General Theory of Relativity (thanks to the perihelion precession of Mercury's orbit), Einstein wanted to apply his field equations to the whole universe and get a model for the same [2]. But he assumed (wrongly) that the universe should be static and unchanging. Of course, Einstein had strong philosophical reason to look for a universe which is static. During that time, there was hardly any observational evidence for an expanding universe.

Not surprisingly he found his equations did not admit a static solution (gravity which is described by GR gives a large scale attraction pulling everything together (collapsing)) unless he added a new term $\Lambda \mathrm{g}_{\alpha \beta}$, giving the more general equation (equation (12)). The $\Lambda$ term essentially introduces a large scale repulsive force $\Lambda r^{2}$ to balance the attractive gravitational force, giving an equilibrium size of a static universe as $a=1 / \sqrt{\Lambda}$. This became known as Einstein's static universe. He estimated its radius as a few billion light years and was happy with it. 
However a few years later, Hubble (using the 100 inch Mt. Wilson telescope near Los Angeles) observed that all far away galaxies show a red shift proportional to distance [8], implying a receding velocity $\propto d$. Hubble's observations definitely suggested an expanding (dynamic) universe with time and not a static one. With the weight of evidence increasing for expansion, Einstein realised that he could have predicted a dynamic model if he had not introduced the $\Lambda$ term. He reportedly remarked later that it was his biggest blunder.

\section{$3 \quad$ Bigger Blunders}

However introduction of $\Lambda$ term was not his real blunder. The biggest blunders were in fact:

1. Not realising that a static universe results only when there is no matter. This was clear even to Newton. The kinetic energy associated with a particle of mass $m$ in the expanding universe is given by, $1 / 2 m \dot{a}^{2}$. If the total mass of the universe is given by $M=4 / 3 \pi a^{3} \rho, \rho$ being the density, then the potential energy associated with the mass $m$ is, $G M m / a$. On equating the two we get

$$
\frac{\dot{a}}{a} \propto \sqrt{\frac{8 \pi G \rho}{3}}
$$

This would imply a static universe, i.e., $\dot{a}=0$, only when $\rho=0$.

2. He thought he got a stable static configuration by balancing the attractive overall gravity with the $\Lambda$ repulsion, thus getting a balancing radius $a_{E} \approx 1 / \sqrt{\Lambda}$ so called Einstein static universe. However it is an elementary problem in physics to check whether the equilibrium (around a position) is stable or unstable, by making small perturbations about the equilibrium coordinate. Einstein did not check for this.

An elementary calculation first pointed out by Eddington[9], and implicitly by Lemaître[10], who found the general solutions of which Einstein's universe was a particular, and clearly unstable, case) shows that Einstein universe is unstable to perturbations. The necessary and sufficient condition for a static solution is, $\dot{a}=\ddot{a}=0$. The density is given by, $\rho=\rho_{0}+\frac{\Lambda c^{2}}{8 \pi G}$, where the second term is the dark energy contribution. To examine stability, we put $a=\Lambda^{-1 / 2}+\delta a$ and $\rho_{0}=\frac{\Lambda}{4 \pi}+\delta \rho_{0}$ into the usual equations

$$
\frac{\dot{a}}{a}=-\frac{4 \pi \rho_{0}}{3}+\frac{1}{3} \Lambda
$$

with, $c=G=1$ in the above equations. So to order $\delta R$, equation (14) becomes

$$
\Lambda^{1 / 2}(\delta \ddot{a})=-\frac{4 \pi}{3} \delta \rho_{0}
$$

As total dust energy is conserved, i.e. $\rho_{0} a^{3}=$ constant, we have

$$
\frac{\delta \rho_{0}}{\rho_{0}}=-3 \frac{\delta a}{a}
$$

Equation (15) then gives

$$
(\delta \ddot{a})-\Lambda \delta a=0
$$

So $\delta a$ grows exponentially in time, with the solution of equation (17) given by

$$
\delta a \propto e^{\sqrt{\Lambda} t}
$$

So the static Einstein universe is unstable. Surprisingly, Einstein did not realise this.

3. What is worse is that he insisted that the Newtonian limit of his extended field equations with $\Lambda$ term is given by Modified Poisson equation given by

$$
\nabla^{2} \phi-\Lambda \phi=4 \pi G \rho
$$

This was a real blunder, which marked an opening statement in a bizarre comedy of errors. Einstein modified Poisson equation would give rise to a potential $\phi=e^{-k r} / r$ falling off with $r$, exponentially, 
rather than a universal $\Lambda r^{2}$ repulsion. This equation is familiar to all physicists through the static version of Yukawa's meson theory which has a spherically symmetric vacuum solution. $\phi=\frac{\text { const }}{r} e^{-r \sqrt{\Lambda}}$.

In the context of Newtonian gravity, such a modification was empirically introduced by Seeliger [11] and Neumann [12] before 1900, to introduce an exponential cut off in the potential to try to explain Mercury's anomalous motion among other things. Einstein was unaware of this work in 1916, but that is not the problem. [13]

It is true that the Poisson equation modified by a term $-\Lambda \phi$ gives rise to an exponential cut-off for gravitational potential. But Einstein's flat assertion that the $\Lambda$ term in his modified field equation has an analogous effect, i.e. leads to a $-\Lambda \phi$ modification of Poisson equation is completely wrong. The correct Newtonian analogue of Einstein's equation with $\Lambda$ term is given by

$$
\nabla^{2} \phi-\Lambda c^{2}=4 \pi G \rho
$$

This indeed gives as a solution a repulsive force $\Lambda r^{2}$, increasing with $r$.

However generations of physicists (even relativity specialists) have repeatedly parroted this mistake of the $\Lambda \phi$ term, rather than the correct $\Lambda c^{2}$ term [14-17]. Some eminent recent examples include:

1. Abraham Pais [18] writes in his magisterial Einstein biography about the analogy between the $\Lambda$ terms in Poisson's equation and Einstein's equations gives exponential cut-off etc.

2. Even Stephen Hawking makes the same mistake. In 1983 Royal Society discussion [19], he begins by saying that Einstein's $\Lambda$ term modifies Poisson equation to give an exponentially falling Neumann type of potential.

3. J Weber in 1989 discussion of weak field approximates with $\Lambda$ term (gravitational waves) has a $e^{-\sqrt{\Lambda} r}$ propagators. [20]

The $\Lambda$ term introduces a force $F=m c^{2}(\Lambda / 3) r$ analogous to a repulsive oscillator potential $\Lambda c^{2} r^{2}$.

This is the correct potential, a force increasing with distance rather than an exponentially decreasing one. Eddington [21] clearly showed this and was one of the few who did not blunder at least in this case. Instead of getting a shielded gravity field, one had now at large distances, almost naked repulsion quite different from Einstein's expected bargain. Incidentally above (major) blunders is not listed in Ohanian's massive tome 'Einstein's mistakes'[22].

\section{Modern Hindsight}

This large scale $\Lambda$ repulsion is the first example of Dark Energy which makes the universe expand faster and faster (accelerating universe). Ironically many of the recent observations [23] are consistent with a $\Lambda$ term, i.e. Einstein's cosmic constant dominating the universe to the extent of $70 \%$.

In 1917 already de Sitter [24] made nonsense of Einstein's static universe (with a $\Lambda$ term) by finding an exact exponentially expanding solution for a Universe with only a $\Lambda$ term and no matter

$$
d s^{2}=-d t^{2}+e^{2 H t}\left(d x^{2}+d y^{2}+d z^{2}\right)
$$

This is also evident from the fact that $\dot{a} / a=$ constant. $\dot{a} / a=\sqrt{\Lambda}=$ constant gives, $d a / a=\sqrt{\Lambda} d t \Rightarrow a=a_{0} e^{\sqrt{\Lambda} t}$. This was immediately after Einstein's paper. In fact this de-Sitter solution forms the seminal basis for modern inflationary universe paradigms. The introduction of the $\Lambda$ term amounts to a redefinition of the vacuum state flat space-time by the de-Sitter space-time. The $\Lambda$ term introduces a negative pressure with an equation of state $P=-\rho c^{2}$ and the negative pressure gives rise to repulsion. This can be seen even in Newtonian context. The acceleration is given by

$$
\begin{aligned}
& \ddot{a}=-\frac{G M}{a^{2}}=-G \frac{4 / 3 \pi a^{3}}{a^{2}}\left(\rho+\frac{3 P}{c^{2}}\right) \\
& \Rightarrow \frac{\ddot{a}}{a}=-\frac{4}{3} \pi G\left(\rho+\frac{3 P}{c^{2}}\right)
\end{aligned}
$$

The negative sign indicates usual gravitational attraction (deceleration). If the pressure is given by $P=-\rho c^{2}$ (as implied by $\Lambda$ ), then $\ddot{a}$ becomes positive (repulsion). Pressure due to a cosmological constant term, $\Lambda$ is 


$$
P_{-v e} \approx \frac{\Lambda c^{4}}{8 \pi G}
$$

The general expression for pressure is

$$
P=w \rho c^{2}
$$

where, $w=-1$ gives cosmological constant $\Lambda$. So a negative pressure implies repulsive gravity. This negative pressure characterises dark energy.

\section{Dark Energy}

Generally one would expect the rate of expansion of the universe to slow down, as once the universe started expanding, the combined gravity of all its constituents should pull it back, i.e. decelerate it. So the deceleration parameter was expected to be a positive value. A negative deceleration parameter $\left(q_{0}\right)$ would imply an accelerating universe, with repulsive gravity and negative pressure. And the measurements of Type Ia supernovae have revealed just that [25,26]. By measuring their flux with redshift, $q_{0}$ is determined to be -0.55 .

Various observations of the dynamics of the universe have implied the dominance of DE. This has led to the introduction of a repulsive gravity source to make the deceleration parameter negative [27]. The dimensionless quantity, deceleration parameter which measures the cosmic acceleration of the universe's expansion is given by

$$
q_{0}=-\frac{\ddot{a} a}{\dot{a}^{2}}
$$

Any bulk motion, whether of a vehicle or any moving object like celestial body, including the universe as a whole is characterised by position $a(t)$ (as a function of time), velocity $(\dot{a}(t)$ ) and acceleration $(\ddot{a}(t))$. The Hubble constant measures rate of expansion $H(a)=\frac{\dot{a}(t)}{a(t)}$. The deceleration parameter is given by

$$
q_{0}=\frac{\Omega_{m}}{2}-\Omega_{\Lambda}
$$

where $\Omega=\rho / \rho_{\mathrm{c}}$ is a dimensionless parameter given by the ratio of the density to the critical density. For dark energy matter, $\Omega_{\overline{\bar{\Lambda}}} \rho_{\Lambda} / \rho_{\mathrm{c}}$, and for matter, $\Omega_{\overline{\bar{m}}} \rho_{m} / \rho_{\mathrm{c}}$, where $\rho_{m}$ is matter density. When the KE term balances the gravitational PE, matter, $\frac{1}{2} m \dot{a}^{2}=\frac{G M m}{a}$, the mass $M=\frac{4}{3} \pi \rho_{a v} a^{3}, \quad \rho_{a v}$ is the average density, then the density corresponding to this condition (critical density) is given as $\rho_{c}=\frac{3 H^{2}}{8 \pi G}$.

The deceleration parameter $q_{0}$ is deduced from luminosity-distance relation (how flux from a distant object falls off with distance) for far away type Ia supernovae in distant galaxies, which is given by

$$
D_{L}=\frac{c z}{H_{0}}\left[1+\frac{\left(1-q_{0}\right)}{2} z\right]
$$

where the flux is equal to $\frac{L}{\left(4 \pi D_{L}^{2}\right)}, L$ is the standard candle luminosity (of the supernova). By measuring flux and with redshift $(z)$ (which is known from wavelength observed) and $H_{0}$ known, $q_{0}$ can be inferred. $q_{0}$ is seen to be negative (-0.55). This together with the fact that $\Omega=\Omega_{m}+\Omega_{\Lambda}=1$ (from CMBR), gives $\Omega_{m} \approx 0.3$ and $\Omega_{\Lambda} \approx 0.7$.

All postulated forms of matter yield a deceleration parameter $q \geq 0$ (positive $q$ ), except in the case of DE. Dark energy dominating the universe at present (accelerating universe) has an equation of state 
given by equation (24), and the current observations do indeed suggest $w$ is close to one. Einstein may turn in his grave because his 'biggest blunder' dominates the universe.

If in future, Dark Energy is confirmed to be just the cosmological constant, Einstein should get two posthumous Nobel Prize, one for gravitational waves prediction and other for the cosmological constant. Einstein's $\Lambda$ may also explain why the universe started expanding in the first place (whereas gravity would have made it collapse instantly). A large cosmic vacuum energy term (predicted in modern unified gauge theories) is equivalent to a (very) large $\Lambda$ term in the early universe, making the universe expand exponentially in the beginning. Negative pressure would create matter so that total energy is zero, in effect, creation ex-nihilo (from nothing), i.e., universe as a free lunch! With improved sensitivity and advanced detection of gravitational waves, then the accurate angle- and frequencydependent response functions of interferometers for gravitational waves arising from various theories of gravity, i.e. general relativity and extended theories of gravity will be the definitive test for general relativity, and help in discriminating among various gravity theories [28].

\section{$6 \quad$ Summary and Conclusion}

But there are a lot of unresolved questions and issues $[29,30]$. Such as, how did such a large vacuum energy drop? And what is the nature of $\Lambda$ ? The non-zero value of $\Omega_{\Lambda}$ has many problems. The value of vacuum density, $\rho_{v a c}=\left(10^{-2} e V\right)^{4}$ is unnaturally small. In quantum field theory, vacuum energy arises from fluctuations of the fields associated with virtual particles. Ironically covariant (i.e. Lorentz invariant) forms of this vacuum (or zero point energy) is of the form, pointed out by Zel'dovich and others, $T_{(\alpha \beta) v a c}=\kappa g_{\alpha \beta}$, which has just the form of the energy-momentum tensor of the $\Lambda$-term. So virtual particles of mass $m$ (created in vacuum), have an energy density $\left(T_{(\mu \nu) v a c}\right)$ of $\sim m^{4} c^{5} / \hbar^{3}$. Particles of mass $m$, separated by $\hbar / m c$ have mass density $m /(\hbar / m c)^{3}$ or energy density $m c^{2} /(\hbar / m c)^{3}=m^{4} c^{5} / \hbar^{3}$. As virtual particles of all masses contribute, we have the Planck mass, giving a large term (e.g. in the very early universe, during quantum gravity era, when particle masses were of this order)

$$
\Lambda_{P l} \sim \frac{m_{P l}^{4} c^{5}}{\hbar^{3}}
$$

This is 120 orders larger than the current density of the universe. Phase transitions in the early universe would lead to release of this vacuum energy (like latent heat) with a density $\sim m^{4} c^{5} / \hbar^{3}$. It is a mystery why the present value is so small, what happened to all these huge vacuum energies. Partial solutions are given in [17] and references therein. The only other length scale is the Hubble radius and coincidentally $\Lambda \sim a_{H}^{-2} \approx 10^{56} \mathrm{~cm}^{-2}$.

Even the electroweak or super-symmetry breaking at TeV scale gives $\rho_{v a c}>(1 T e V)^{4}$, the observed value is $10^{54}$ times smaller. At present $\Omega_{m}$ (scaling as $a^{-3}$ ) and $\Omega_{\Lambda}$ (scaling as $a^{0}$ ) are of same order of magnitude, implying we live in a very special era. Why is the vacuum density $(\Lambda)$ density, comparable to matter density at present epoch?

Answers to the old problems raise new problems with no answers. Einstein neither liked $\Lambda$, nor the then formulation of quantum theory. Ironically, it is now believed that quantum fluctuations (quantum vacuum energy) should generate $\Lambda$ (as discussed above) and perhaps even Einstein gravity.

\section{References}

1. P. Kerszberg, The Invented Universe: The Einstein-De Sitter Controversy (1916-17) and the Rise of Relativistic Cosmology. Clarendon Press, Oxford, 1989.

2. A. Einstein, "Cosmological Considerations in the General Theory of Relativity (English translation)." Sitz. König. Preuss. Akad., part 1, p. 142, http://einsteinpapers.press.princeton.edu/vol6-trans/433, 1917. 
3. A. Einstein, The Meaning of Relativity. Routledge, London, 2003.

4. A. Einstein, "The Foundation of the General Theory of Relativity (English translation)." Annalen der Physik, vol. 49, p. 769, http://einsteinpapers.press.princeton.edu/vol6-trans/158, 1916.

5. C. Sivaram, K. Arun and O. V. Kiren, 100 years of Einstein's Theory of Relativity: An Introduction to Gravity and Cosmology. Ane Books, New Delhi, 2016.

6. A. Einstein, (1905) Annalen der Physik 322, 891; M. Saha, The Principle of Relativity: Original Papers by A. Einstein and H. Minkowski (English translation), University of Calcutta, pp. 1-34, 1920.

7. J. Wheeler and K. Ford, Geons, Black Holes, and Quantum Foam. W.W. Norton \& Company, New York, 2000.

8. E. Hubble, "A relation between distance and radial velocity among extra-galactic nebulae." Proc. Natl. Acad. Sci. U.S.A., vol. 15, pp. 168-173, 1929.

9. A. S. Eddington, "On the Instability of Einstein's Spherical World." Mon. Not. Roy. Astron. Soc., vol. 90, pp. 668-678, 1930.

10. G. Lemaître, "Expansion of the universe, A homogeneous universe of constant mass and increasing radius accounting for the radial velocity of extra-galactic nebulae." Mon. Not. Roy. Astron. Soc., vol. 91, pp. 483-490, 1931.

11. H. von Seeliger, "On Newton's law of gravitation." Pop. Astron., vol. 5, pp. 544-551, 1898.

12. C. Neumann, Allgemeine Untersuchung über das Newtonsche Prinzip der Fernwirkungen. Teubner, Leipzig, 1896.

13. C. O'Raifeartaigh, M. O'Keeffe, W. Nahm and S. Mitton, "Einstein's 1917 Static Model of the Universe: A Centennial Review." The European Physical Journal H, vol. 42, pp. 431-474, 2017.

14. C. Sivaram, "Mond, dark matter and the cosmological constant." Astrophys. Space Sci., vol. 219, pp. 135-141, 1994.

15. C. Sivaram, "A non-anthropic origin for a small cosmological constant." Bull. Astron. Soc. India, vol. 27, pp. 377-382, 1999 .

16. C. Sivaram, "On Zero-Point Fluctuations, the Cosmological Constant, and the Graviton Mass." Astrophys. J., vol. 520, pp. 454-455, 1999.

17. C. Sivaram, "A non-anthropic origin for a small cosmological constant." Mod. Phys. Lett. A, vol. 14, pp. 23632366, 1999 .

18. A. Pais, Subtle is the Lord: The Science and the Life of Albert Einstein. Oxford University Press, Oxford, 1982.

19. S. W. Hawking, "The cosmological constant." Philos. Trans. R. Soc. Lond. A, vol .310, pp. 303-310, 1983.

20. J. Weber, Gravitational antenna bandwidths and cross sections. In: Schutz, B.F., Ed., Gravitational Wave Data Analysis, NATO ASI C253, Kluwer, Dordrecht, pp. 195-200, 1989.

21. A. S. Eddington, The Mathematical Theory of Relativity. Cambridge University Press, Cambridge, 1923.

22. H. C. Ohanian, Einstein's Mistakes: The Human Failings of Genius. W.W. Norton \& Company, New York, 2008.

23. P. A. R. Ade et al., "Planck 2013 results. XVI. Cosmological parameters." Astron. Astrophys., vol. 571, p. A16, 2014.

24. W. de Sitter, "On the relativity of inertia. Remarks concerning Einstein's latest hypothesis." Proc. Roy. Neth. Acad. Arts and Sci., vol. 19, pp. 1217-1225, 1917.

25. A. G. Riess et al., "Observational Evidence from Supernovae for an Accelerating Universe and a Cosmological Constant." Astron. J., vol. 116, pp . 1009-1038, 1998.

26. S. Perlmutter et al., "Measurements of $\Omega$ and $\Lambda$ from 42 High-Redshift Supernovae." Astrophys. J., vol. 517, pp. 565-586, 1999.

27. M. Jones and R. Lambourne, An Introduction to Galaxies and Cosmology. Cambridge University Press, Cambridge, 2004.

28. C. Corda, "Interferometric detection of gravitational waves: the definitive test for General Relativity." Int. J. Mod. Phys. D, vol. 18, pp. 2275-2282, 2009.

29. K. Arun, K., S. B. Gudennavar and C. Sivaram, "Dark matter, dark energy, and alternate models: A review." Adv. Space Res., vol. 60, pp. 166-186, 2017.

30. K. Arun, S. B. Gudennavar, A. Prasad and C. Sivaram, "Alternate models to dark energy." Adv. Space Res., vol. 61, pp. 567-570, 2018. 